\title{
CONDICIONANTES PERSONALES Y AMBIENTALES DE LA CONDUCTA AUTODETERMINADA EN LA ADOLESCENCIA CON ALTERACIONES DEL DESARROLLO INTELECTUAL
}

\section{PERSONAL AND ENVIRONMENTAL DETERMINANTS OF SELF-DETERMINED BEHAVIOR IN ADOLESCENTS WITH INTELLECTUAL DEVELOPMENTAL DISORDERS}

\author{
Manoel Baña Castro \\ Luisa Losada Puente \\ Jesús Miguel Muñoz Cantero
}

Departamento de Filosofía y Métodos de Investigación en Educación, Universidad de A Coruña. España

\begin{abstract}
Resumen: El interés reciente por la calidad de vida de las personas con alteraciones del desarrollo resulta importante para la toma de decisiones y el diseño de los programas de atención, desarrollando recursos, pautas y estrategias así como criterios relevantes para la provisión diferencial de recursos y apoyos. Un buen predictor de la calidad de vida es la autodeterminación que busca desarrollar estrategias centradas en la persona y sus necesidades desde una perspectiva integral para su vida y desarrollo. Los avances en la comprensión y el desarrollo de modelos teóricos y prácticas orientadas a la promoción de la autodeterminación evidencian la importancia de conocer el efecto de los condicionantes individuales y ambientales sobre la adquisición y expresión de las habilidades que permiten a la persona actuar como agente causal de su vida. El objetivo del presente trabajo es analizar los resultados de las principales investigaciones que estudian el papel de dichos condicionantes sobre la adquisición y expresión de la autodeterminación en adolescentes con discapacidad intelectual. Se emplea la modalidad de investigación documental para el estudio de documentos de los últimos quince años publicados en las principales bases de datos de educación y de psicología. Los resultados evidencian la repercusión y correlación de múltiples factores sobre el aprendizaje y la expresión de autodeterminación. Se discuten los resultados y su impacto en el diseño de programas de instrucción en autodeterminación en la adolescencia.
\end{abstract}

Palabras Clave: autodeterminación; alteraciones del desarrollo intelectual; variables predictoras; características individuales; influencias ambientales

\begin{abstract}
The growing interest in the quality of life of people with developmental disorders is important for decision making and designing care programs, developing resources, guidelines, strategies and relevant criteria for differential provision of resources and support. A good predictor of quality of life is self-determination which seeks to develop strategies focused on the person and their needs from a holistic perspective for life and development. Advances in the understanding and the development of theoretical models and practices aimed at promoting self-determination demonstrate the importance of knowing the effect of individual and environmental conditions on the acquisition of skills that enable a person to act as a causative agent of their life. The aim of this study is to analyze the results of major research studying the role of these conditions on the acquisition and expression of self-determination in adolescents with intellectual disabilities. Documentary research is used for the study of documents of the past fifteen years published in leading databases of education and psychology. The results show the impact and correlation of multiple factors on learning and expression of self-determination. The results and their impact on the design of training programs in self-determination in adolescence are discussed.
\end{abstract}

Key Words: self-determination; intellectual developmental disorders; predictor variables; individual characteristics; environmental influences

\section{Introducción}

En las últimas décadas, el énfasis en promoción de la autodeterminación en personas con alteraciones del desarrollo intelectual ha motivado intensos debates entre expertos en la esfera internacional acerca de su fundamenta- ción (Arellano y Peralta, 2013; Verdugo, 2009 Walker et al., 2011). Cabe matizar, que, cuando nos referimos al concepto de alteraciones o trastornos del desarrollo intelectual, atendemos a la definición propuesta por la American Psychiatric Association (APA, 2014) como "un trastorno que comienza durante el período de

Correspondencia: Manoel Baña Castro. Director de la Unidad Clínico-Asistencial de Trastronos del Espectro del Autismo y Trastornos del Desarrollo. Correo Electrónico: mbcastro@udc.gal 
desarrollo y que incluye limitaciones en el funcionamiento intelectual como también del comportamiento social en los dominios conceptual, social y práctico" (p. 17). En la línea de esta definición, la investigación desarrollada hasta el momento en materia de autodeterminación ha puesto en evidencia los presupuestos de determinismo filosófico acerca la causalidad de los elementos externos y leyes naturales sobre la conducta humana y se ha centrado en demostrar la interconexión de múltiples factores y elementos de carácter biológico, ambiental y personal (Wehmeyer, 2006b).

De esta forma, la autodeterminación es definida como la disposición de cualidades y habilidades adquiridas por instrucción directa a lo largo de la vida, que permiten a la persona actuar como agente causal de su propia vida. Se trata de un constructo adquirido por la persona a lo largo de su vida y en interacción con su entorno, facilitado por un proceso de enseñanzaaprendizaje estructurado, y condicionado por elementos internos a la persona y aquellos que son propios del contexto en el que se desarrolla, así como por la oportunidades que éste le facilita para actuar de forma autónoma, autorregulada, intencional, controlada y con conocimiento de las propias capacidades y limitaciones (Arellano \& Peralta, 2013; Peralta, 2007; Rojas, 2004; Wehmeyer, 2006b).

El reconocimiento del carácter multifactorial de la conducta autodeterminada contrapone la pasividad y la dependencia de la persona con discapacidad, reclamando para ella la toma de control, la interdependencia y la disponibilidad de apoyos y de entornos múltiples y variados que se configuren atendiendo a sus necesidades específicas en cada contexto de su vida (personal, familiar, escolar, laboral, etc.) (AreIlano \& Peralta, 2013; Rojas, 2004; Vega et al., 2013; Wehmeyer, 2006b).

El principio de autodeterminación se sitúa, de este modo, como un referente a nivel científico y profesional en la provisión de servicios para personas con discapacidad, y como un estándar en la lucha contra la opresión social gestada desde los medios de comunicación, las fundaciones benéficas, los profesionales de diferentes ámbitos, la política en general o las formas de producción, así como para la defensa de la capacidad de decidir y opinas de estas personas (Arellano y Peralta, 2013;
Walker et al., 2011). Junto a otros conceptos como la emancipación, los derechos y la dignidad, la autoestima, la eficacia y la autoeficacia, percepción de la valía y calidad de vida, la autodeterminación se introduce progresivamente en la investigación y en los servicios que se ofrecen a las personas con discapacidad.

Respecto al ámbito científico, la preocupación de los expertos se ha centrado en romper con los presupuestos del determinismo filosófico acerca de la causalidad de los elementos externos o leyes naturales que rigen la conducta humana, y en demostrar la interconexión de múltiples factores y elementos de carácter biológico, ambiental y personal. De hecho, multitud de modelos teóricos dan cuenta de la complejidad de este concepto, y que refiere a uno o varios de estos elementos en sus explicaciones (Ryan \& Deci, 2000; Field \& Hoffmann, 1996, 2002; Wehmeyer, 1996, 1999, 2006a).

Nos referimos, en específico, a Wehmeyer $(1996,1999)$ quien formula un modelo sustentador de constructo de autodeterminación, denominado el Modelo Funcional de la Autodeterminación, dotando a este constructo de una completa definición y operativización en cuatro elementos: autonomía, autorregulación, capacitación psicológica (empowerment) y autorrealización, los cuales se ponen en funcionamiento en la medida en el que la persona: (a) adquiera, por instrucción directa a lo largo de su vida las habilidades y cualidades que le permitan expresar conductas autodeterminadas observables; (b) inicie y responda esas conductas de forma volitiva, autónoma, independiente y autorregulada; (c) actúe como agente causal de su vida y de su entorno; (d) enriquezca su conducta en la interacción con el ambiente y a través de la influencia de los factores personales mediadores en su aprendizaje.

Tomando como base éste y otros modelos teóricos de la autodeterminación (Modelo en cinco componentes de Field, Martin, Miller, Ward \& Wehmeyer, 1998; Field \& Hoffman, 1996, 2002; Modelo Ecológico Tripartito de Abery \& Stancliffe, 1996, 2003a,b; entre otros) así como los presupuestos formulados por el paradigma de los apoyos, que propone dotar a las personas con discapacidad de los apoyos individualizados y adaptados a sus necesidades, preferencias e intereses partiendo de los principios de autodeterminación e inclusión (Baña, 2011; Thompson et al., 2003; Verdugo, 2009), la atención priori- 
taria de los servicios y de los profesionales que trabajan con personas con discapacidad se ha volcado sobre el estudio de la necesidades de apoyo requeridas con el fin de proveerlas de los medios y recursos necesarios para alcanzar los resultados personales deseados y la mejora de su calidad de vida.

Además, surge un creciente número de publicaciones que versan sobre la autodeterminación y sus repercusiones sobre la calidad de vida de estas personas, lo que justifica nuestro interés por mejorar la comprensión de este amplio y complejo concepto analizando la repercusión de las características personales y el entorno en que se desarrolla el individuo sobre la vida de los adolescentes con discapacidad intelectual y del desarrollo.

Por todo ello, hoy en día es reconocida la confluencia de múltiples factores y variables sobre la consecución de una mayor autodeterminación en las diversas etapas de la vida. La asunción de este presupuesto ha llevado a numerosos investigadores e investigadoras a analizar, a nivel teórico y empírico, el papel mediador de los diversos condicionantes individuales y contextuales sobre el desarrollo de la autodeterminación (véase Tabla 1).

La autodeterminación no solo puede ser explicada por la influencia de las características biológicas, genéticas o personales ni por el impulso de factores externos, leyes naturales o fuerzas ajenas a la persona sino que resulta de la confluencia de múltiples predictores o antecedentes que condicionan el aprendizaje y expresión de la autodeterminación o, bien, surgen como efecto de su manifestación por parte de la persona.

Al asumirse la agencia causal de toda conducta (Wehmeyer \& Litlle, 2013) se reconoce a la persona la capacidad de tomar decisiones de forma intencionada y libre de interferencias expertas; empero, esto no supone considerar que las personas hagan las cosas por sí mismas, sino que actúen como catalizadoras de los acontecimientos de su vida (Wehmeyer, Palmer, Agran, Mithaug, \& Martin, 2000) o, lo que es lo mismo, que se conviertan en "colaboradores activos o 'autores' de su comportamiento" (Walker et al., 2011, p.7). Pero además, este comportamiento se encuadra dentro de un contexto social (la conducta determinada por uno mismo o por el otro); es, precisamente, en su interacción con el ambiente donde el individuo aprende y aplica su capacidad para ser responsable y protagonista de sus actuaciones.

Por todo ello, este estudio analiza el papel mediador de los condicionantes individuales y del ambiente sobre la adquisición y desarrollo de la conducta autodeterminada. Se plantea como objetivo estudiar las principales investigaciones desarrolladas en los últimos años, acerca de la influencia de los condicionantes individuales y ambientales en la conducta autodeterminada de las personas con discapacidad intelectual y otras alteraciones del desarrollo, atendiendo especialmente a la etapa de la adolescencia y la transición a la vida adulta.

Tabla 1

Condicionantes de la conducta autodeterminada

\begin{tabular}{|c|c|c|}
\hline & & Variables \\
\hline \multirow{5}{*}{ Factores internos } & & Edad \\
\hline & Biológicos y genéticos & Género \\
\hline & & Funcionamiento intelectual \\
\hline & Conductuales y de la & Conducta adaptativa \\
\hline & personalidad & Valores, creencias y actitudes \\
\hline \multirow{8}{*}{ Factores externos } & Contextuales & Entorno próximo (vivienda) \\
\hline & & Cultura/ étnia \\
\hline & & Entorno escolar/laboral \\
\hline & & Estatus socioeconómico \\
\hline & Familiares & Estilos de educación parental \\
\hline & & Conocimiento, valores y actitudes familiares \\
\hline & & Escolarización (tipo de centro) \\
\hline & Escolares & $\begin{array}{l}\text { Prácticas pedagógicas y actitudes del profesorado } \\
\text { Posibilidades y deseo de participación }\end{array}$ \\
\hline
\end{tabular}




\section{Método}

El estudio emplea la modalidad de investigación documental encuadrada dentro de la metodología de tipo cualitativo. Con esta modalidad de estudio se posibilita el regreso al pensamiento original de los estudiosos sobre el tema, ofreciendo así mayor rigor a las fuentes originales mediante la exploración de diversas e interconectadas interpretaciones de un mismo suceso (Gómez, 2011; Hodder, 2012).

\section{Materiales}

El material empleado en esta investigación son las fuentes primarias y secundarias que registran información acerca de la mediación de los factores individuales y ambientales sobre la autodeterminación, extraídos de seis bases de datos nacionales e internacionales de las áreas de educación y psicología: CSIC ISOC - Ciencias Sociales y Humanidades; DIALNET; ERIC; PsycINFO; SCOPUS; Web Of Science, WOS.

\section{Procedimiento}

El procedimiento de recogida y posterior análisis de dicha información se realiza siguiendo un esquema en cuatro pasos (Bernette, 2013; Monroy \& Hernández, 2014):

(a) Formulación del problema: especificación de objetivos y finalidad del estudio, identificación $\mathrm{del} / \mathrm{de}$ los contexto/s interrelacionados objeto de estudio y definición de criterios para la selección de los documentos.

(b) Extracción de los datos: selección de un criterio de búsqueda; priorización del interés suscitado en el contexto de investigación; descripción de tipo de documentos de interés; selección de una teoría que sustenta a la investigación.

(c) Análisis e interpretación de los resultados, en base a un proceso en dos fases:

- Fase descriptiva: codificación de la información más relevante y clasificación de los códigos en categorías de análisis, descritas y analizadas teóricamente para su mejor comprensión (Flick, 2014).

-Fase interpretativa: comprensión de las relaciones entre variables relevantes, en base a las categorías propuestas, y comparación de los códigos y categorías principales en cada caso artículo.

(d) Presentación de las conclusiones.
La búsqueda en bases de datos se desarroIla introduciendo uno o varios de los siguientes términos en español y en inglés: autodeterminación (o self-detemination), factores (o factors); factores ambientales (o environmental factors) y discapacidad (o disability), con la que son extraídos más de doscientos documentos. Éstos son filtrados empleando descriptores relacionados con los conceptos de autodeterminación, discapacidad, educación especial, dificultades de aprendizaje, ambiente, características individuales, edad, funcionamiento intelectual, conducta adaptativa, habilidades sociales, actitud del estudiante, entorno próximo, vivienda, educación especial, familias, etc.

Se eliminan los artículos con referencias a la autodeterminación como derecho político o colectivo, en relación con la salud y con el deporte, aquellos que no se refieren a la autodeterminación como constructo global y las revisiones teóricas cuyo foco de atención no sean los factores que afectan al desarrollo de la conducta autodeterminada. Se emplean los filtros de las bases de datos para excluir documentos referentes a áreas de economía y empresa, medicina, bioquímica y biología genética y molecular y enfermería. Finalmente, se introduce un filtro temporal, eliminando aquellas investigaciones anteriores al año 2000.

La selección de textos da como resultado un total de veinte documentos de los últimos quince años, seleccionados por interés y relevancia de sus títulos y resúmenes en los que se recogen las aportaciones que evidencian la influencia de las características personales (edad, sexo, funcionamiento intelectual) así como desde los entornos más globales, cuya influencia afecta de modo menos directo a la persona (la cultura), hasta aquellos que repercuten directamente sobre la persona, relacionados con las características del entorno en que se desenvuelve la persona, con el estatus socioeconómico y los estilos de educación familiar y con la escolarización y participación en el proceso de transición a la vida adulta.

\section{Resultados}

El análisis documental ha permitido atender a los principales condicionantes individuales y ambientales considerados mediadores o predictores de la autodeterminación en personas con discapacidad intelectual. A continuación, se presentan las principales consideraciones de 
algunos de ellos; concretamente, analizaremos el papel del funcionamiento intelectual, la edad, el género, la cultura, la familia y la escuela.

\section{Condicionantes individuales}

Nivel de inteligencia o funcionamiento intelectual. Las primeras investigaciones en el campo de la discapacidad sostenían que el coeficiente intelectual $(\mathrm{Cl})$ determinaba las posibilidades de la persona con discapacidad para actuar de forma autodeterminada y, por tanto, reconocían que las limitaciones en el número y la complejidad de habilidades que el individuo podía ejecutar suponían un freno a su capacidad para actuar como agente causal de su vida. Desde hace décadas, se reconoce que la alteración cognitiva no está reñida con la capacidad de la persona para alcanzar la plena autodeterminación (Bissoto, 2014; Wehmeyer, 2006a; Wehmeyer et al., 2011).

La teoría de la modificabidad cognitiva reconoce que todo individuo tiene capacidad y dicha capacidad es modificable, dependiendo de la disposición de la estimulación adecuada al potencial de aprendizaje del individuo (Baña, 2011). Diversos estudios analizan la relación entre autodeterminación y $\mathrm{Cl}$ corroborando, en su mayoría, la existencia de una fuerte correlación entre ambos, pero matizando que la inteligencia no tiene por qué ser un factor determinante ni predictor de la conducta autodeterminada (Chambers et al., 2007; Gómez-Vela, Verdugo, González-Gil, Badía, \& Wehmeyer, 2010; Gómez-Vela, Verdugo, González-Gil, Badía, \& Wehmeyer, 2012; Lee et al., 2012; Vega et al. 2013; Wehmeyer \& Gardner, 2003).

\section{Edad}

La autodeterminación es un proceso que se sucede en un continnum a lo largo de la vida, por medio de instrucción directa y, cuyo punto álgido se produce en la adolescencia, momento en el que la persona comienza a emplear sus aprendizajes en la práctica de su vida diaria (Bissoto, 2014; Contini, Cohen Imach, Coronel, \& Mejail, 2012; Gómez-Vela et al., 2010; Peralta, 2008, citado en Vega García et al., 2013; Wehmeyer, 2013).

Se ha constatado la estrecha relación entre mayor autodeterminación y mejores resultados en la adultez para la juventud con discapacidad así como mayores problemas de inadaptación, aislamiento, cambios de humor y problemas comunicativos en la adultez, en jóvenes con menores niveles de autodeterminación (Nota, Ferrari, Soresi y Wehmeyer, 2007; Shogren, Wehmeyer, Palmer, \& Paek, 2013; Vega García et al., 2013; Wehmeyer, 1996; Wehmeyer \& Palmer, 2003, citado en Nota et al., 2007).

\section{Género}

Los presupuestos del enfoque centrado en la igualdad de género postulan que, más allá del desarrollo de las características sexuales relacionadas con la pubertad, no deberían existir diferencias entre hombres y mujeres en cuanto a la consecución de un mayor nivel de autodeterminación. Lo cierto es que no existen, todavía, datos concluyentes acerca de tales diferencias (Wehmeyer, 2006a; Wehmeyer et al., 2011), pues la información es limitada y, en su mayoría, contradictoria.

Ejemplos de tales discordancias se pueden observar en los resultados que nos ofrecen los estudios de Gómez-Vela, Verdugo et al. (2012), Lee et al. (2012), Nota et al. (2007), Shogren, Wehmeyer, Palmer y Paek (2013) o Wehmeyer y Gardner (2003).

\section{Condicionantes ambientales}

Cultura. Desde la Teoría de la Autodeterminación se asume que las necesidades a nivel competencial, de autonomía y de interacción social son innatas, universales y compatibles, y su cumplimiento favorece el correcto funcionamiento de todos los individuos en las diversas culturas y sociedades (Hui y Tsang, 2012). Ahora bien, pese a la universalidad de este constructo, su aprendizaje y expresión, así como la importancia y utilidad conferida por cada individuo puede variar en función de la cultura de pertenencia (Shogren et al., 2007). La cultura de cada lugar, como comportamiento aprendido y compartido, modela las percepciones, las formas de comportamiento y el sentido de la realidad de cada persona (Wehmeyer et al., 2011).

El interés por indagar sobre la influencia del factor cultural sobre la expresión de la autodeterminación es todavía incipiente, encontrando tan solo un limitado número de publicaciones que demuestran la existencia de diferencias en los niveles de autodeterminación en función de la cultura (Frankland et al., 2004, citado en 
Wehmeyer et al., 2011; Leake \& Boone, 2007; Trainor, 2005; Zhang, 2005; Zhang, Landmark, Grenwelge \& Montoya, 2010) con resultados muy dispares y poco concluyentes.

Familia. La discapacidad de un hijo o hija es un acontecimiento que afecta al conjunto familiar y puede llegar a generar una serie de efectos adversos (altos niveles de estrés, bienestar general más negativo, relaciones sociales más pobres, sentimientos de crianza negativos, peor satisfacción entre cónyuges, mayor carga económica y de cuidados, etc.).

El grueso de la investigación sobre la influencia de la familia en la conducta autodeterminada de los hijos e hijas se centra en el análisis del estatus socioeconómico y los estilos educativos parentales revelando la existencia de una compleja red de relaciones entre estatus, educación parental y autodeterminación. Se evidencia la existencia de una correlación positiva entre mayores ingresos familiares y una mejor enseñanza en habilidades y capacidades vinculadas a la autodeterminación (Zhang, 2005); la influencia de la cultura de origen familiar sobre la predisposición a ofrecer mayores oportunidades a los hijos para dar sus opiniones y elegir (Leake \& Boone, 2007), así como de las paulas educativas parentales (autoritaria, democrática o permisiva) sobre la expresión de preferencias, establecimiento de metas o toma de decisiones (Wehmeyer et al., 2011).

Así mismo, se ha indagados sobre las percepciones, creencias y actitudes familiares hacia la promoción de la autodeterminación de sus hijos con alteraciones del desarrollo intelectual. Los estudios revelan la influencia de las percepciones y creencias familiares como barreras o facilitadores a la adquisición y expresión de habilidades relacionadas con la autodeterminación en los hijos/as (Carter, Trainor, Owens, Sweden, \& Sun, 2009; Chambers et al., 2007); el impacto de la predisposición de los padres a que sus hijos decidan sobre aspectos importantes en sus vidas (Zhang, 2005); y la problemática derivada de la sobreprotección parental y la resistencia a que los hijos se enfrenten a situaciones complejas en su vida (Trainor, 2005)

Escuela. La autodeterminación se sitúa, según Ryan (2000, citado en Huertas, 2012) como la necesidad de hacer algo por uno mismo; en el contexto educativo, esto equivale a ofrecer al alumnado de nuevos retos y dificultades que el alumnado debe afrontar, dotándoles de una sensación de competencia y de responsabilidad sobre la propia decisión tomada acerca de la ejecución de la tarea.

Diversas investigaciones encuentran niveles superiores en autodeterminación y mayor autonomía y conocimiento sobre si mismos en el alumnado con discapacidad intelectual escolarizado en centros específicos (Shogren, Wehmeyer, Palmer, \& Paek, 2013; Vega García et al., 2013) o integrado en aulas específicas (Cho, Wehmeyer, \& Kingston, 2012; Zhang, 2001, citado en Shogren et al., 2013), donde el punto central de la educación se orienta a la adquisición de habilidades relacionadas con la autonomía, la independencia, la resolución de problemas cotidianos, etc. frente a tareas puramente vinculadas a los contenidos académicos que se ejecutan dentro de las escuelas ordinarias.

Respecto a las prácticas pedagógicas, una buena parte del profesorado comprende la importancia de la autodeterminación como un aspecto esencial en el proceso educativo de todo el alumnado (Cho et al., 2012; GómezVela et al., 2012), destacando sus beneficios para la consecución de una mayor inserción y mejor calidad de vida. No obstante, esta positiva percepción del profesorado acerca de las posibilidades de sus estudiantes varía en función del grado de discapacidad de cada alumno, percibiéndose distorsionado el trato recibido por el alumnado con discapacidades más graves o severas por parte del profesorado. El profesorado que trabaja a alumnado con discapacidad leve es más proclive a considerar importante la formación del alumnado en autodeterminación y su propia formación e información sobre cómo fomentar la conducta autodeterminada en su alumnado, que aquellos que trabajan con alumnado con discapacidad severa (Cho et al., 2012; Shogren et al., 2013; Wehmeyer, 2006b).

Y en relación con las posibilidades que se le ofrece al alumnado para involucrarse en la vida escolar, se evidencia la importancia de la participación del alumnado en el desarrollo de la autodeterminación y, si bien es confuso el carácter predictor de este factor (Shogren et al., 2007; Shogren et al., 2013), se ha demostrado su relación con la obtención de resultados positivos en la adultez (Wehmeyer, Palmer, Soukup, Gardner \& Laurence, 2008; Wehmeyer, Palmer, Shogren, William-Diehm \& Soukup, 2010). 
En definitiva, tanto los apoyos y las expectativas, como las oportunidades que se les ofrece a estos jóvenes para participar en la vida escolar y comunitaria, son componentes esenciales de las intervenciones con este alumnado. De hecho, existen evidencias empíricas que apoyan la eficacia de las intervenciones a través de programas de instrucción en autodeterminación en la adolescencia que toman como eje central maximizar el control individual, fomentar su independencia y autonomía, ejercitar su capacidad para la toma de decisiones y la resolución de problemas y las cuales, además, adoptan un enfoque global, que involucra no solo al adolescente, sino también a su entorno familiar, escolar y comunitario (Arellano \& Peralta, 2013; Field et al., 1998, Lee et al., 2012, Peralta, 2007; Thompson et al., 2003).

\section{Discusión}

Este trabajo se centra en analizar algunos de los principales factores involucrados en la adquisición y expresión de las habilidades relacionadas con la conducta autodeterminada. Si bien el análisis se ha ocupado de la revisión de publicaciones acerca de la autodeterminación de la adolescencia con discapacidad en general, lo cierto es que varios estudios centrados en abordarlas características individuales evidencian la relación entre funcionamiento intelectual y autodeterminación aunque su carácter predictor ha quedado en entredicho. Se infiere de ellos la complejidad de la relación entre autodeterminación e inteligencia donde, si bien se admite la influencia del nivel de inteligencia o grado de discapacidad sobre la capacidad de la persona para ser autodeterminada, queda demostrado que la discapacidad de la persona no es determinante de su posibilidad de alcanzar las habilidades requeridas para ser agente causal de su propia vida.

En relación al género, los resultados continúan siendo poco concluyentes. Se cuestiona, por tanto, si las variaciones halladas en las investigaciones analizadas pueden deberse a los cambios acontecidos en los últimos años en relación a la instrucción en autodeterminación de los hombres y las mujeres con discapacidad y si tales discrepancias puede justificarse por errores muestrales de algunos de los estudios.

Y en relación con la edad, queda demostrada la incidencia de este condicionante en la expresión de la autodeterminación así como la importancia de estimular desde tempranas edades las habilidades conducentes al desarrollo de un comportamiento autodeterminado.

Por su parte, los estudios que versan sobre los factores ambientales mediadores de la conducta autodeterminada concluyen la influencia de un entorno estimulante que favorezca la adquisición y expresión de comportamientos autodeterminados así como la relevancia que tienen las pautas educativas parentales y la percepción de los padres y madres acerca de las capacidades de sus hijos.

Igualmente, se evidencia la correlación entre el tipo de prácticas pedagógicas y actitudes del profesorado y la consecución de mayores niveles de autodeterminación, al igual que ocurre con las oportunidades que se les ofrezcan a estos jóvenes de experimentar en situaciones naturales, de poner a prueba sus capacidades, de reconocer sus limitaciones y de equivocarse y acertar.

\section{Conclusiones}

El estudio de estos factores contribuye, por un lado, a ampliar la comprensión acerca de su impacto sobre la construcción y la expresión de la conducta autodeterminada y, por otro lado, a justificar las actuaciones que se enfocan hacia la promoción de la plena autodeterminación de las personas.

Para impulsar la autodeterminación es necesario conocer los factores y variables que inciden y afectan a la misma lo que ha de determinar las prácticas y su orientación para la educación de las personas con alteraciones del desarrollo intelectual. La autodeterminación no es un planteamiento exclusivo de estas personas pero supone una perspectiva esencial en el desarrollo y aprendizaje de las mismas. El cambio alude a la centración de la persona como protagonista de su educación acorde a los últimos avances científicos como una de las claves para la mejora de las prácticas educativas. Los apoyos o ayudas individuales que necesita cada alumno deben ser evaluados con la máxima precisión y revisados con frecuencia por los equipos profesionales. Sin olvidar, que junto a la importancia asignada a los conocimientos académicos debe también prestarse gran atención a otras dimensiones del comportamiento del individuo que se manifiestan a través del modelo de calidad de vida. 
Tomar decisiones, expresar preferencias, y tener conciencia y confianza en sí mismo lleva consigo múltiples experiencias en la educación y en la vida que son independientes del nivel o grado de alteración en el desarrollo intelectual que pueda poseer. No obstante, pese a los avances de las últimas décadas en materia de autodeterminación, continúan existiendo ciertas lagunas en el estudio de los factores subyacentes al desarrollo y expresión de la autodeterminación, por lo que indagar y analizar en sus relaciones va suponer estrategias de compromiso, cambio y acción que conlleven el desarrollo de la autodeterminación en todas las personas, incluidas las que poseen una alteración del desarrollo intelectual.

Por tanto, se hace necesario continuar la investigación en esta línea, de forma que contribuya a la mejor comprensión de los elementos que afectan al desarrollo de la conducta autodeterminada en cada etapa del desarrollo y que nos permitan guiar las prácticas que favorezcan su adquisición en personas con discapacidad intelectual.

\section{Referencias}

Abery, B. \& Stancliffe, R. (1996). The ecology of selfdetermination. En D.J. Sands \& M.L. Wehmeyer (Eds.). Self-determination across the life span (pp. 111-145). Baltimore: Paul H. Brookes.

Abery, B. \& Stancliffe, R. (2003b). A tripartite-ecological theory of self-determination. En M.L. Wehmeyer, B.H. Abery, D.E. Mithaug, \& R.J. Stancliffe (Eds.). Theory in self-determination. Foundations for educational practice (pp. 43-78). Springfield: Charles C. Thomas.

Abery, B. \& Stancliffe, R. (2003a). An ecological theory of self determination. En M.L. Wehmeyer, B.H. Abery, D.E. Mithaug \& R.J. Stancliffe (Eds.), Theory in selfdetermination. Foundations for educational practice (pp. 25-42). Springfield: Charles C. Thomas.

American Psychiatric Association (2014). Diagnostic and statistical manual of mental disorders ( $5^{\mathrm{a}}$ ed.) (1 ${ }^{\mathrm{a}}$ Reimpresión). Washington: Autor.

Arellano, A. \& Peralta, F. (2013). Autodeterminación de las personas con discapacidad intelectual como objetivo educativo y derecho básico: estado de la cuestión. Revista Española de Discapacidad, 1(1), 97-117. doi.: 10.5569/2340-5104.01.01.05

Baña Castro, M. (2011). Los trastornos del espectro autista y generales del desarrollo: inclusion social y calidad de vida. Ciencias Psicológicas, 5(2), 183-191.

Bernette García, F. (2013). Análisis de contenido. En A.L. Marín y A. Noboa (Coord.), Conocer lo social: estrategias y técnicas de construcción y análisis de datos (pp.193-225). Madrid: Fragua.

Bissoto, M.L. (2014). Deficiência intelectual e processos de tomada de decisão: estamos enfrentando o desafio de educar para a autonomia?. Educação Unisinos, 18(1), 3-12. doi.: 10.4013/edu.2014.181.01
Carter, E.W., Trainor, A., Owens, L., Sweden, B. \& Sun, Y. (2009). Self-determination prospects of youth with high-incidence disabilities: divergent perspectives and related factors. Journal of Emotional and Behavioral Disorders, 18(2), 67-81. doi: 10.1177/1063426609332605

Chambers, C.R., Wehmeyer, M.L., Saito, Y., Lida, K.M., Lee, Y., \& Singh, V. (2007). Self-determination: What do we know? Where do we go?. Exceptionally, 15(1), 3-15. doi: 10.1080/09362830709336922

Cho, H.-J., Wehmeyer, M.L., \& Kingston, N.M. (2012). The effect of social and classroom ecological factors on promoting self-determination in elementary school. Preventing School Failure: Alternative Education for Children and Youth, 56(1), 19-28. doi: 10.1080/1045988X.2010.54419

Contini, E.N., Cohen Imach, S., Coronel, C.P., \& Mejail, S. (2012). Agresividad y retraimiento en adolescentes. Ciencias Psicológicas, 7(1), 17-28.

Field, S. \& Hoffman, A. (1996). Steps to self-determination: A curricula to help adolescents learn to achieve their goals. Austin: Pro-Ed.

Field, S. \& Hoffman, A. (2002). Preparing youth to exercise self-determination: quality indicators of schools environments that promote the acquisition of knowledge, skills, and beliefs related to selfdetermination. Journal of Disability Policy Studies, 3(2), 114-119. doi: 10.1177/10442073020130020701

Field, S., Martin, J., Miller, R., Ward, M., \& Wehmeyer, M. (1998). Self-determination for persons with disabilities: a position statement of me division career development and transition. Career Development and Transition for Exceptional Individuals, 21(2), 113-128. doi: 10.1177/088572889802100202

Flick, U. (2014). An introduction to qualitative research (5th ed.). Londres: Sage.

Gómez, L.E. (2011). Un espacio para la investigación documental. Revista Vanguardia Psicológica, 1(2), 226-233. Recuperado de: http://umb.edu. co:82/revp/index.php/vanguardiapsicologica/article/ viewArticle/36

Gómez-Vela, M., Verdugo, M.A., Badía, M., González-Gil, F., \& Calvo, M.I. (2010). La escala de evaluación de la autodeterminación: un instrumento en desarrollo. En M.A. Verdugo, M. Crespo, \& T. Nieto (Coords.), Aplicación del paradigma de calidad de vida. VII Seminario de Actualización Metodológica en Investigación sobre Discapacidad (pp. 83-90). Salamanca: Publicaciones del INICO.

Gómez-Vela, M., Verdugo, M.A., González-Gil, F., Badía, M., \& Wehmeyer, M.L. (2012). Assessment of selfdetermination of Spanish students with intellectual and other educational needs. Journal of Applied Research in Intellectual Disabilities, 23(5), 505-506. Recuperado de: www.ncbi.nlm.nih.gov/pmc/articles/PMC4104407

Hodder, I. (2012). The interpretation of documents and material culture. En J. Goodwin (Ed.), Sage biographical research (Vol. 1-4) (pp. 171-188) . doi: 10.4135/9781446268537

Huertas, J.A. (2012). Los efectos de los afectos en la motivación y la autorregulación. Ciencias Psicológicas, 6(1), 45-55.

Hui, E.K.P. \& Tsang, S.K. (2012). Self-determination as a psychological and positive youth development construct. The Scientific World Journal, 2012, 1-7. doi: 10.1100/2012/759358 
Leake, D. \& Boone, R. (2007). Multicultural perspectives on self-determination from youth, parent, and teacher focus group. Career Development for Exceptional Individuals, 30(2), 104-115. doi: 10.1177/08857288070300020101

Lee, Y., Wehmeyer, M, Palmer, S., William-Diehm, K., Davies, D., \& Stock, S. (2012). Examining individual and instruction-related predictors of the selfdetermination of students with disabilities: multiple regression analysis. Remedial Special Education, 33(3), 150-161. doi: 10.1177/0741932510392053

Monroy, F. \& Hernández Pina, F. (2014). Factores que influyen en los enfoques de aprendizaje universitario. Una revisión sistemática. Educación XXI, 17(2), 105124. doi: 10.5944/educxx1.17.2.11481

Nota, I., Ferrari, L., Soresi, S., \& Wehmeyer, M.L. (2007). Self-determination, social abilities and the quality of life of people with intellectual disabilities. Journal of Intellectual Disability Research, 51(11), 850-865. doi: 10.1111/j.1365-2788.2006.00939.x

Peralta, F. (2007). La autodeterminación de las personas con discapacidad intelectual como meta educativa. En M. Liesa, P. Allueva \& M. Puyuelo (Coords.), Educación y acceso a la vida adulta en personas con discapacidad (pp.263-277). Barbasto: Fundación Ramón J. Sender.

Rojas Pernia, S. (2004). Autodeterminación y calidad de vida en personas discapacitadas. Experiencia desde un hogar de grupo (Tesis doctoral). Universitat Autónoma de Barcelona. España. Recuperado de: http://www.tdx.cat/bitstream/handle/10803/5044/ srp1de2.pdf?sequence $=1$

Ryan, R.M. \& Deci, E.L. (2000). Self-determination theory and the facilitation of intrinsic motivación, social development, and well-being. American Psichologist, 55(1), 68-78. doi.: 10.1037110003-066X.55.1.68

Shogren, K.A., Wehmeyer, M.L., Palmer, S.B., \& Paek, Y. (2013). Exploring personal and school environment characteristics that predicts self-determination. Exceptionality: a Special Education Journal, 21(3). doi: 10.1080/09362835.2013.802231

Shogren, K.A., Wehmeyer, M.L., Palmer, S.B., Soukup, J.H., Little, T.D., Garner, N., \& Lawrence, M. (2007). Examining individual and ecological predictors of the self-determination of students with disabilities. Exceptional Childen, 73(4), 488-509. Recuperado de: eirc.ed.gov/?id=EJ817517

Thomson, J.R., Hughes, C., Schalock, R.I., Silverman, W., Tassé, M.J., Bryant, B., Craig, E.M. ,\& Campbell, E.M. (2003). Integrando los apoyos en la evaluación y la planificación. Siglo Cero, 34(2), 36-55. Recuperado de: http://riberdis.cedd.net/handle/11181/3062

Trainor, A. (2005). Self-determination perceptions and behaviors of diverse students with LD during the tran sition planning process. Journal of Learning Disabilities, 38(3), 233-248. Recuperado de: http:// www.ncbi.nlm.nih.gov/pubmed/15940961

Vega García, C., Gómez Vela, M., Fernández Pulido, R., \& Badía Corbella, M. (2013). El papel del contexto educativo en la autodeterminación. Análisis de su influencia en el proceso de transición a la vida adulta de alumnos con discapacidad intelectual. Revista Iberoamericana de Educación, 63, 19-33. Recuperado de: http://riberdis.cedd.net/bitstream/ handle/11181/4359/EI papel_del contexto educativo. pdf? sequence $=\overline{1} \& \mathrm{rd}=00 \overline{3} 13 \overline{1} 131195957 \overline{8}$
Verdugo, M.A. (2009) Buenas prácticas para la mejora de la calidad de vida y autodeterminación del alumnado con necesidades específicas de apoyo. Murcia: Diversa.

Verdugo, M.A., Canal, R., Jenaro, C., Badia, M., \& Aguado, A.(2012). Integración en la Comunidad. Salamanca: Publicaciones del Inicio.

Walker, H.M., Calkins, C., Wehmeyer, M.L., Walker, L., Bacon, A., Palmer, S.B.,... Jonhson, D.R. (2011). A socialecological approach to promote self-determination. Exceptionaly: A Special Education Journal, 19(1), 6-18. doi: 10.1080/09362835.2011.537220

Wehmeyer, M.L. (1996). Self-determination as an educational outcome: Why is it important to children, youth and adults with disabilities?. En D.J. Sands \& M.L. Wehmeyer (Eds.). Self-determination across the life span: independence and choice for people with disabilities (pp. 15-34). Baltimore: Paul H. Brookes.

Wehmeyer, M.L. (1999). A functional model of selfdetermination: describing development and implementing instruction. Focus on Autism and Other Developmental Disabilities, 14(1), 53-61. Recuperado de http://www.imdetermined.org/files_images/general/ SD5_A\%20Functional\%20Model\%20of.pdf

Wehmeyer, M.L., Palmer, S.B., Agran, M., Mithaug, D.E., \& Martin, J.E. (2000). Promoting causal agency. The serlfdetermined learning model of instruction. Exceptiona Children, 66(4), 439-453. Recuperado de: http://www.jennyhatchjusticecenter.org/sites/default/ files/promoting_causal_agency_self-determined_ learning_model_instruction.pdf

Wehmeyer, M.L. \& Gardner, N.W. (2003). The impact of personal characteristics of people with intellectual and developmental disability on self-determination and autonomus functioning. Journal of Applied Reseach in Intellectual Disabilities, 16, 255-265. doi: 10.1046/j.1468-3148.2003.00161.x

Wehmeyer, M. (2006a). Autodeterminación y personas con discapacidades severas. Siglo Cero, 37(4), 5-16. Recuperado de: http://www.feaps.org/comunicacion/ documentos/siglo_220.pdf

Wehmeyer, M. (2006) $)$. Factores intraindividuales y medioambientales que afectan a la autodeterminación. Siglo Cero, 37(4), 47-56. Recuperado de: http://sid. usal.es/idocs/F8/ART9355/articulos4.pdf

Wehmeyer, M.L., Palmer, S.B., Soukup, J.H., Garner, N.W., \& Lawrence, M. (2008). Self-determination and student transition planning knowledge and skills: predicting involvement. Exceptionally: $A$ Special Education Journal, 15(1), 31-44. doi: 10.1080/09362830709336924

Wehmeyer, M.L., Palmer, S.B., Shogren, K., WilliamsDiehm, K., \& Soukup, J.H. (2010). Establishing a causal relationship between intervention to promote self-determination and enhanced student selfdetermination. Journal of Special Education, 46(4), 195-210. doi: 10.1177/0022466910392377

Wehmeyer, M.L., Abery, B.H., Zhang, D., Ward, K., Willis, D., Hossain Amin, W., ... Walker, H. (2011). Personal selfdetermination and moderating variables that impact efforts to promote self-determination. Exceptionally, 19(1), 19-30. Recuperado de: www.aucd.org/docs/ ScalingUpPaper2Final.pdf

Wehmeyer, M. \& Little, T. (2013). Self-determination. En M. Wehmeyer, (ed.), The Oxford handbook of positive psychology and disability (pp. 116-136). Nueva York, EE.UU.: Oxford University Press. 
Zhang, D. (2005). Parent practices in facilitating selfdetermination skill: the influences of cultura, socioeconomic status, and children's special education status. Research and Practice for Persons with Severe Disabilities, 20(2), 154-162. doi: 10.2511/ rpsd30.3.154
Zhang, D., Landmark, L., Grenwelge, C. \& Montoya, L. (2010). Culturally diverse parents' perspective on self-determination. Education and Training in Autism and Developmental Disabilities, 45(2), 175-186. Recuperado de: http://eric.ed.gov/?id=EJ884372

Para citar este artículo:

Baña Castro, M., Losada Puente, L., \& Muñoz Cantero, J. M. (2015). Condicionantes personales y ambientales de la conducta autodeterminada en la adolescencia con alteraciones del desarrollo intelectual. Ciencias Psicológicas, 9(2), 283 - 292. 